\title{
A quick and easy-to-use clinical scale to assess balance in Parkinson's disease
}

This article was published in the following Dove Press journal:

Journal of Parkinsonism \& Restless Legs Syndrome

29 November 2012

Number of times this article has been viewed

\section{Abraham Lieberman' \\ Sara Dhanani' \\ Rohit Dhall' \\ Di $\operatorname{Pan}^{2}$}

'Muhammad Ali Parkinson Center (MAPC) of Barrow Neurological Institute, St Joseph's Hospital and Medical Center, Phoenix, AZ, USA; ${ }^{2}$ Center for Health Information and Research, Arizona State University, Tempe, AZ, USA
Correspondence: Abraham Lieberman Muhammad Ali Parkinson Center of Barrow Neurological Institute, St Joseph's Hospital and Medical Center, Phoenix, AZ 850I3, USA

Email abedoc@gmail.com
Background: Balance difficulty is a major contributor to falls in Parkinson's disease (PD). However, the new Movement Disorder Society Unified Parkinson's Disease Rating Scale (MDS-UPDRS) motor examination contains only one subtest - postural instability - and it is only $3 \%$ of the total that assesses balance. There are several balance scales, but they were not developed for PD and they do not complement the MDS-UPDRS. There is a need for a quick and easy-to-use balance scale in PD and one that complements the MDS-UPDRS.

Methods: The Barrow Neurological Institute (BNI) balance scale was developed as a complement to the MDS-UPDRS and was used to evaluate the risk of falling in 53 PD patients. There were nine patients who fell three times per month ("fallers"). The BNI scale was compared with the widely used Tinetti scale. The Tinetti scale has 16 subtests; the BNI scale has five.

Results: The nine fallers had a significantly longer PD duration, a higher MDS-UPDRS score, a higher (worse) BNI balance score, and a lower (worse) Tinetti score compared to 44 nonfallers. Fallers were significantly more impaired on the MDS-UPDRS subtest of postural stability and on the BNI scale. A BNI scale score $\geq 13$ had a sensitivity of $100 \%$ and a specificity of $88.6 \%$ in identifying fallers, whereas a Tinetti scale score $\leq 18$ had a sensitivity of $55.6 \%$ and a specificity of $95.5 \%$ in identifying fallers. The BNI scale with five subtests is easier to perform than the Tinetti with 16 subtests.

Conclusion: The BNI balance scale is more sensitive and specific in predicting falls than the Tinetti. It is a useful adjunct to the MDS-UPDRS.

Keywords: balance, falls, Parkinson's disease, Tinetti scale

\section{Introduction}

Falls are a common and serious complication of Parkinson's disease (PD). ${ }^{1-3}$ In a study of 761 PD patients who were admitted to hospital, only 114 patients (15\%) were admitted for management of their PD, whereas 251 patients $(33 \%)$ were admitted because of falls. ${ }^{4}$ In another study of 124 PD patients who were admitted to hospital, 79 patients (64\%) were admitted for falls. ${ }^{5}$ Given the relatively high percentage of falls in patients with PD and the seriousness of the falls, there is an interest in identifying patients who will fall ("fallers"), and among fallers identifying those tests that predict falls.

The Unified Parkinson's Disease Rating Scale (UPDRS) is the most utilized test for evaluating patients with PD. The original UPDRS has a question in the activities of daily living, on falls. ${ }^{6}$ This question is absent on the revised Movement Disorder Society UPDRS (MDS-UPDRS). ${ }^{7}$ Both the original UPDRS and the MDS-UPDRS have only one subtest on balance (postural instability) - the "pull test." This subtest 
makes up only 4 points out of a total of 132 points, ie, only $3.0 \%$ of the motor examination - a small part for assessing such an important problem.

There are several scales for assessing balance, of which the Tinetti ${ }^{8,9}$ is arguably the most widely used. The Tinetti scale consists of tests of balance (16 points) and tests of gait (12 points). There are 16 subtests of the Tinetti. Patients who score $\leq 18$ on the combined balance and gait components of the Tinetti are considered at high risk for falling. Despite this scale being widely used to assess fall risks, it was not designed to accommodate the specific balance impairments observed in PD and it duplicates many subsets of the MDSUPDRS. Similar issues can be raised about the other balance tests. ${ }^{10,11}$ There are more sophisticated balance and gait tests, ${ }^{12-14}$ but they require special instruments and training. We believe there is a need for a balance test that is quick and easy to perform and that complements the MDS-UPDRS.

Based on the above, we developed the Barrow Neurological Institute (BNI) balance scale, which focuses on PD and addresses issues that result in falls in PD, such as turning, ${ }^{15-17}$ or standing on one foot while reaching or walking up or down stairs, ${ }^{18,19}$ and walking in confined spaces. ${ }^{20,21}$ The BNI balance scale does not duplicate any part of the MDSUPDRS, and thus can be used as a complement to it. The BNI balance scale, with five subtests, was compared to the Tinetti scale, with 16 subtests.

\section{Methods}

We examined 53 consecutive patients with a diagnosis of PD. Patients with a Mini-Mental Status Examination score $<27$, symptomatic orthostatic hypotension, neuropathy, myelopathy, cerebellar dysfunction, atypical Parkinson's including multiple system atrophy, progressive supranuclear palsy, cortico-basilar degeneration, normalpressure hydrocephalus, and extensive white-matter disease. While eight patients (15\%) were on benzodiazepines, anticholinergics (including amantadine), or antidepressants, none of the fallers were on these drugs.

All patients completed the experiences of daily living part of the revised UPDRS and were examined utilizing the motor portion of the MDS-UPDRS, the Hoehn and Yahr (HY) scale, the Tinetti, and with the BNI balance scale by two of the authors (AL and SD). The BNI balance scale incorporates subtests relevant for $\mathrm{PD}$ that are related to falls, of which turning and standing on one leg are subtests of the Tinetti (item 8 of the balance section) and Berg (items 11 and 14) scales. Examiners had previously demonstrated proficiency on the UPDRS motor examination, the
Tinetti scale, the Berg Balance Scale, and tandem walking. Specific interobserver reliability testing using kappa statistics was not performed on the components of the Tinetti, the Berg, or tandem walking. Some of these components comprise the BNI balance scale, including standing on one foot, tandem gait, and turning.

All patients were asked about falls using the BNI balance question. ${ }^{22}$ Attention was paid to the circumstances of the fall: turning, freezing of gait, standing on one foot while reaching for an object. Patients who had had at least three falls during the month prior to the visit were considered to be fallers. All patients on levodopa/carbidopa were examined during the "medication-on" state. All patients were informed that the information on their evaluation could be used for research, but that they personally could not be identified. Approval for publishing our evaluation of the 53 PD patients was obtained from the institutional review board at St Joseph's Hospital and Medical Center.

\section{$\mathrm{BNI}$ balance scale}

\section{Turning (8 points)}

Patients were asked to turn $360^{\circ}$. First, they turned to the right (4 points). After completing this, they turned to the left (4 points). Scoring was similar to the Berg, except 4 is normal on the Berg and 0 is normal on the BNI.

0: normal; $360^{\circ}$ turn accomplished in $\leq 4$ seconds (usually in four steps)

1: $360^{\circ}$ turn accomplished in $>4$ seconds (usually in five or six steps)

2: $360^{\circ}$ turn accomplished safely but slowly (usually in seven or eight steps)

3: $360^{\circ}$ turn accomplished, but patient needs close supervision (usually in nine or ten steps)

4: needs assistance in turning (or can't turn).

\section{Standing on one foot (8 points)}

Scoring was similar to the Berg, except 4 is normal on the Berg and 0 is normal on the BNI. Standing on right foot alone, 4 points; standing on left foot alone, 4 points.

0 : able to lift leg independently and hold for $>10$ seconds (with no assistance or support)

1: able to lift leg independently and hold for 5-10 seconds (with no assistance or support)

2: able to lift leg independently and hold for $\geq 3$ seconds (with no assistance or support)

3: able to lift leg independently and hold for $<3$ seconds (may require assistance or support)

4: unable to try. 


\section{Tandem gait (4 points)}

Patients were asked to take ten steps, placing one foot in front of the other, the heel of one foot touching the toe of the other.

0: can walk ten steps, with no missteps (deviation from the midline)

1: can walk ten steps, with one misstep

2: can walk ten steps, with two, three, or four missteps

3: can walk ten steps, with five or more missteps; usually, patient cannot take five or more consecutive steps

4: cannot tandem walk.

There were nine patients who had fallen at least three times in the month prior to the visit (ie, fallers). There were 44 patients who had not fallen or had fallen less than three times in that month (nonfallers). The following were compared between fallers and nonfallers: age; duration of PD; MDS-UPDRS motor score (maximum 132 points); axial MDS-UPDRS motor score (maximum 24 points, consisting of the sum of the scores for arising from a chair, gait, freezing of gait, postural stability, the "pull test," posture, and body bradykinesia); Tinetti test (28 points); and BNI balance test (maximum 20 points).

Continuous variables such as age, disease duration, HY stage, MDS-UPDRS motor score, axial MDS-UPDRS motor score, BNI scale score, and Tinetti score were analyzed using $t$-tests (Table 1). Categorical variables obtained by categorizing postural stability, and the BNI subtests, were analyzed using chi-square tests (Table 2). The categorical variables were used as identifiers to construct a logistic regression model to predict falls. The sensitivities and specificities were calculated for each logistic model and

Table I Comparison of mean values of patients' age, disease duration, disease stage, Movement Disorder Society Unified Parkinson's Disease Rating Scale (MDS-UPDRS) motor and axial scores, Barrow Neurological Institute (BNI) balance score, and Tinetti score, between nonfallers (44 patients) and fallers (nine patients) with a critical level of $P<0.05$

\begin{tabular}{lccl}
\hline Variables & $\begin{array}{l}\text { Nonfallers } \\
\text { (44 patients) }\end{array}$ & $\begin{array}{l}\text { Fallers } \\
\text { (9 patients) }\end{array}$ & P-value \\
\hline Age & $69.9 \pm 7.9$ & $69.4 \pm 3.4$ & 0.7802 \\
Duration & $5.0 \pm 2.5$ & $9.2 \pm 3.8$ & $<0.0001$ \\
Hoehn and Yahr Scale & $2.36 \pm 0.67$ & $3.50 \pm 0.61$ & $<0.0001$ \\
stage & & & \\
UPDRS score & $18.07 \pm 8.00$ & $33.78 \pm 17.17$ & 0.0257 \\
UPDRS axial score & $5.18 \pm 3.19$ & $10.89 \pm 4.37$ & $<0.0001$ \\
BNI balance score & $6.20 \pm 3.30$ & $13.77 \pm 2.04$ & $<0.0001$ \\
Tinetti score & $23.61 \pm 3.23$ & $18.22 \pm 4.18$ & $<0.0001$ \\
\hline
\end{tabular}

compared. The SAS 8.01 statistical software package (SAS Institute, Cary, NC) was used for statistical analyses.

\section{Results}

The comparison of continuous variables between fallers and nonfallers revealed that fallers had longer disease duration, higher HY stage score, and higher MDS-UPDRS motor score and axial score than that of nonfallers. Fallers were not older than nonfallers, suggesting that PD severity and not age was a factor related to falls in these patients. Also, fallers had a higher (worse) BNI balance score and a lower (worse) Tinetti score than nonfallers. The differences in all the continuous variables except the age were statistically significant at $P<0.05$. In half of the patients, upon careful questioning of the patient and caregiver, it was able to be determined that falls occurred while turning, while trying to stand or standing on one leg and reaching for an object, while maneuvering in a crowded room, or while walking up or down stairs.

Comparison of categorical variables revealed that all the fallers had MDS-UPDRS postural stability scores $\geq 2$, BNI standing on one foot scores $\geq 3$, BNI turning scores $\geq 3$, and BNI tandem-gait scores $\geq 2$, compared to $50.0 \%, 52.3 \%, 43.2 \%$, and $38.6 \%$, respectively, in the case of nonfallers. For the Tinetti score, $55.6 \%$ of fallers had a score of $\leq 18$ (considered at risk of falls), whereas only $4.6 \%$ of nonfallers had a score of $\leq 18$. All these differences between fallers and nonfallers were statistically significant. There were too few patients with freezing of gait during the examination to compare this variable between fallers and nonfallers.

To compare the performance of the Tinetti scale with the BNI balance scale in identifying risk of falls, two logistic models were constructed using Tinetti and BNI balance scores as independent variables and their sensitivity and specificity were calculated (Table 3 ). Using the Tinetti scale at a threshold of $\leq 18$, falls were predicted at a sensitivity of $55.6 \%$ with a high specificity of $95.5 \%$, whereas the BNI balance scale (at a score $\geq 13$ ) had $100 \%$ sensitivity with $88.6 \%$ specificity. Moreover, the ability of MDS-UPDRS postural stability subtest, BNI balance scale subtests, and combination of postural stability subtest and all the subtests of BNI balance scale in predicting falls were also explored (Table 4).

\section{Discussion}

Balance difficulty is different from gait difficulty in PD. ${ }^{23-30}$ This is the basis for the development of the BNI balance 
Table 2 Comparison of fallers and nonfallers utilizing categorical variables such as Movement Disorder Society Unified Parkinson's Disease Rating Scale (MDS-UPDRS) postural stability score, Barrow Neurological Institute (BNI) balance scale subset scores

\begin{tabular}{llll}
\hline Variables & $\begin{array}{l}\text { Nonfallers } \\
\text { (44 patients) }\end{array}$ & $\begin{array}{l}\text { Fallers } \\
\text { (9 patients) }\end{array}$ & P-value \\
\hline UPDRS - postural stability $\geq 2$ & $50.0 \%$ & $100.0 \%$ & 0.0055 \\
BNI - standing on one foot $\geq 3$ & $52.3 \%$ & $100.0 \%$ & 0.0076 \\
BNI - turning $\geq 3$ & $43.2 \%$ & $100.0 \%$ & 0.0019 \\
BNI - tandem walking $\geq 2$ & $38.6 \%$ & $100.0 \%$ & 0.0008 \\
\hline
\end{tabular}

Note: Comparisons were made utilizing chi-square statistics at a critical level of $P<0.05$.

question and the BNI balance scale. Balance difficulty is a major factor in falls, and falls are a major problem in PD. The problem of balance difficulty and falls is one that has not received the attention it merits, and it certainly has not received the attention it merits on the UPDRS. The newly developed BNI balance question and the BNI balance scale, with its focus on balance difficulty, will, we believe, be a useful complement to the MDS-UPDRS.

Once a patient begins to fall, and the falls cannot be attributed to mishaps or accidents (walking on a slippery floor, tripping over an object, or being pushed or jostled), the patient and family take protective measures, such as providing the patient with a walker or wheelchair and/or observing the patient closely. Such measures minimize the frequency and severity of falls but do not eliminate them. Patients continue to fall when transferring from a wheelchair, when walking with a walker and inadvertently lifting up the walker when turning, or when walking up or down stairs. How does one record falls in such situations? In this study, a patient is considered to have fallen when the patient stumbles and his/her hands or knees touch the ground (falling forward), when a patient's lower back touches the floor (falling backward), or when a patient staggers and his/her back touches a wall or chair.

In this study, fallers had more advanced PD than nonfallers: they had longer PD duration and had higher scores on the MDS-UPDRS motor examination, the axial part of the MDS-UPDRS motor examination, and the HY scale. Fallers, however, were not older than nonfallers, implying that PD severity and not age was a factor in their falls. The MDS-UPDRS and the HY scale (stage level $<3$ ) do

Table 3 Comparison of the Tinetti scale and the Barrow Neurological Institute (BNI) balance scale in predicting falls in Parkinson's disease

\begin{tabular}{lll}
\hline Balance scales & Sensitivity & Specificity \\
\hline Tinetti scale score $\leq 18$ & $55.6 \%$ & $95.5 \%$ \\
BNI balance scale & $100.0 \%$ & $88.6 \%$ \\
score $\geq 13$ & & \\
\hline
\end{tabular}

not predict falls reliably, ${ }^{20}$ hence the need for an additional instrument, and we believe that the BNI scale is such an instrument.

The BNI scale is easier to use than the Tinetti. It has five subtests - turning to the right, turning to the left, standing on the right foot, standing on the left foot, and tandem walking - none of which duplicate any of the subtests on the MDS-UPDRS. The Tinetti has 16 subtests, some of which duplicate subtests on the MDS-UPDRS. The sensitivity of the BNI is much greater than the Tinetti $(100 \%$ vs $55.6 \%)$, but the specificity is slightly less ( $88.6 \%$ vs $95.5 \%)$. The BNI scale is complementary to the MDS-UPDRS. The BNI balance scale combined with the MDS-UPDRS pull test is especially useful in identifying patients who are at risk for falling.

The limitations of the BNI scale are: (1) that it was compared with the Tinetti in a relatively small number of patients (53) with a small number of fallers (9); (2) the BNI balance scale has not been tested in a non-Parkinson's population; (3) while examiners were proficient in the UPDRS motor examination, the Tinetti, the Berg, and the tandem-gait scales, a test of interobserver reliability was not performed on the specific subtests of the BNI scale - subtests such as turning, standing on one leg, and tandem gait - that are also subtests of the Tinetti, the Berg, and the tandem-gait scale. With the above shortcomings, we nonetheless believe that the BNI balance scale combined with the MDS-UPDRS will also be useful in following patients who are having balance difficulty and are undergoing treatment for their difficulty,

Table 4 Prediction of falls utilizing specific categorical variables from the Movement Disorder Society Unified Parkinson's Disease Rating Scale (MDS-UPDRS) and the Barrow Neurological Institute (BNI) balance scale

\begin{tabular}{lll}
\hline Categorical variables & Sensitivity & Specificity \\
\hline UPDRS - postural stability $\geq 2$ & $100.0 \%$ & $50.0 \%$ \\
BNI - standing on one foot $\geq 2$ & $100.0 \%$ & $47.7 \%$ \\
BNI - turning $\geq 2$ & $100.0 \%$ & $56.8 \%$ \\
BNI - tandem walking $\geq 2$ & $100.0 \%$ & $61.4 \%$ \\
\hline
\end{tabular}


including physical and occupational therapy, drug therapy, and deep-brain stimulation.

\section{Disclosure}

This study was part of the office practice of the authors. There was no sponsorship, and the authors report no conflicts of interest in this work.

\section{References}

1. Michalowska M, Fiszer U, Krygowska-Wajs A, Owczarek K. Falls in Parkinson's disease. Causes and impact on patients' quality of life. Funct Neurol. 2005;20(4):163-168.

2. Matinolli M, Korpelainen JT, Sotaniemi KA, Myllyla VV, Korpelainen R. Recurrent falls and mortality in Parkinson's disease: a prospective two-year follow-up study. Acta Neurol Scand. 2011;123(3):193-200.

3. Grimbergen YA, Munneke M, Bloem BR. Falls in Parkinson's disease. Curr Opin Neurol. 2004;17(4):405-415.

4. Temlett JA, Thompson PD. Reasons for admission to hospital for Parkinson's disease. Intern Med J. 2006;36(8):524-526.

5. Schrag A, Ben-Shlomo Y, Quinn N. How common are complications of Parkinson's disease? J Neurol. 2002;249(4):419-423.

6. Fahn S, Marsden CD, Calne DB, Goldstein M, editors. Unified Parkinson's Disease Rating Scale. Florham Park: Macmillan Health Care Information; 1987.

7. Goetz CG, Tilley BC, Shaftman SR, et al. Movement Disorder Society-sponsored revision of the Unified Parkinson's Disease Rating Scale (MDS-UPDRS): scale presentation and clinimetric testing results. Mov Disord. 2008;23(15):2129-2170.

8. Tinetti ME. Performance-oriented assessment of mobility problems in elderly patients. J Am Geriatr Soc. 1986;34(2):119-126.

9. Tinetti ME, Williams TF, Mayewski R. Fall risk index for elderly patients based on number of chronic disabilities. Am J Med. 1986;80(3):429-434.

10. Berg KO, Wood-Dauphinee SL, Williams JI, Maki B. Measuring balance in the elderly: validation of an instrument. Can J Public Health. 1992;83 Suppl 2:S7-S11.

11. Powell LE, Myers AM. The activities-specific balance confidence (ABC) scale. J Gerontol A Biol Sci Med Sci. 1995;50A(1):M28-M34.

12. Wallmann HW. Comparison of elderly nonfallers and fallers on performance measures of functional reach, sensory organization, and limits of stability. J Gerontol A Biol Sci Med Sci. 2001; 56(9):M580-M583.

13. Shumway-Cook A, Woollacott, M. Motor Control: Translating Research into Clinical Practice. Philadelphia: Lippincott Williams \& Wilkins; 2007.

14. Means KM. The obstacle course: a tool for the assessment of functional balance and mobility in the elderly. J Rehabil Res Dev. 1996;33(4): 413-429.
15. Stack EL, Ashburn AM, Jupp KE. Strategies used by people with Parkinson's disease who report difficulty turning. Parkinsonism Relat Disord. 2006;12(2):87-92.

16. Crenna P, Carpinella I, Rabuffetti M, et al. The association between impaired turning and normal straight walking in Parkinson's disease. Gait Posture. 2007;26(2):172-178.

17. Mak MK, Patla A, Hui-Chan C. Sudden turn during walking is impaired in people with Parkinson's disease. Exp Brain Res. 2008;190(1):43-51.

18. Morris M, Iansek R, Smithson F, Huxham F. Postural instability in Parkinson's disease: a comparison with and without a concurrent task. Gait Posture. 2000;12(3):205-216.

19. Jacobs JV, Horak FB, Tran VK, Nutt JG. Multiple balance tests improve the assessment of postural stability in subjects with Parkinson's disease. J Neurol Neurosurg Psychiatry. 2006;77(3):322-326.

20. Bloem BR, Grimbergen YA, Cramer M, Willemsen M, Zwinderman AH. Prospective assessment of falls in Parkinson's disease. J Neurol. 2001;248(11):950-958.

21. Ebersbach G, Gunkel M. Posturography reflects clinical imbalance in Parkinson's disease. Mov Disord. 2011;26(2):241-246.

22. Lieberman A, Krishnamurthi N, Dhall R, et al. A simple question about falls to distinguish balance and gait difficulties in Parkinson's disease. Int $J$ Neurosci. Epub August 21, 2012.

23. Hely MA, Morris JG, Reid WG, Trafficante R. Sydney Multicenter Study of Parkinson's disease: non-L-dopa-responsive problems dominate at 15 years. Mov Disord. 2005;20(2):190-199.

24. Nova IC, Perracini MR, Ferraz HB. Levodopa effect upon functional balance of Parkinson's disease patients. Parkinsonism Relat Disord. 2004;10(7):411-415.

25. Gervais-Bernard H, Xie-Brustolin J, Mertens P, et al. Bilateral subthalamic nucleus stimulation in advanced Parkinson's disease: five year follow-up. J Neurol. 2009;256(2):225-233.

26. Krack P, Batir A, Van Blercom N, et al. Five-year follow-up of bilateral stimulation of the subthalamic nucleus in advanced Parkinson's disease. N Engl J Med. 2003;349(20):1925-1934.

27. Moro E, Lozano AM, Pollak P, et al. Long-term results of a multicenter study on subthalamic and pallidal stimulation in Parkinson's disease. Mov Disord. 2010;25(5):578-586.

28. Rodriguez-Oroz MC, Obeso JA, Lang AE, et al. Bilateral deep brain stimulation in Parkinson's disease: a multicentre study with 4 years follow-up. Brain. 2005;128(Pt 10):2240-2249.

29. Visser JE, Allum JH, Carpenter MG, et al. Subthalamic nucleus stimulation and levodopa-resistant postural instability in Parkinson's disease. J Neurol. 2008;255(2):205-210.

30. Koller WC, Glatt S, Vetere-Overfield B, Hassanein R. Falls and Parkinson's disease. Clin Neuropharmacol. 1989;12(2):98-105.
Journal of Parkinsonism \& Restless Legs Syndrome

\section{Publish your work in this journal}

Journal of Parkinsonism and Restless Legs Syndrome is an online, open access, peer-reviewed journal. The journal publishes review articles, historical reviews, original research articles, case reports, letters to the editor, clinical teaching cases, neuroradiology highlights, neuropathology highlights, neuropsychiatry highlights, autobiographies, conference

\section{Dovepress}

proceedings, abstracts and book reviews. The manuscript management system is completely online and includes a very quick and fair peerreview system, which is all easy to use. Visit http://www.dovepress.com/ testimonials.php to read real quotes from published authors. 